\title{
The Performance of Double Bootstrap Method for Large Sampling Sequence
}

\author{
Muhamad Safiih Lola, Nurul Hila Zainuddin* \\ School of Informatics and Applied Mathematics, University Malaysia Terengganu, Kuala Nerus, Malaysia \\ Email: safiihmd@umt.edu.my, *hila.zainuddin@gmail.com
}

How to cite this paper: Lola, M.S. and Zainuddin, N.H. (2016) The Performance of Double Bootstrap Method for Large Sampling Sequence. Open Journal of Statistics, 6, 805-813.

http://dx.doi.org/10.4236/ojs.2016.65066

Received: July 22, 2016

Accepted: October 15, 2016

Published: October 18, 2016

Copyright $\odot 2016$ by authors and Scientific Research Publishing Inc. This work is licensed under the Creative Commons Attribution International License (CC BY 4.0).

http://creativecommons.org/licenses/by/4.0/

\begin{abstract}
Studies on the iteration procedure in double bootstrap method have given a great impact on confidence interval performance. However, the procedure was claimed to be complicated and demand intensive computer processor. Considering this problem, an alternative procedure was proposed in this research. Despite of using small sampling sequence, this research was aimed to increase the accuracy estimation using a second replication number which resulted in a large sampling sequence of double bootstrap. In this paper, the alternative double bootstrap method was hybrid onto an example model and its performance was based on Studentised interval. The performance was examined in simulation study and real sample data of sukuk Ijarah. The result showed that hybrid double bootstrap model gave more accurate estimation in terms of its shorter length when dealing with various parameter values and has shown to improve the single bootstrap estimation.
\end{abstract}

\section{Keywords}

Double Bootstrap, Confidence Interval, Sampling Sequence, EWMA, Sukuk Ijarah

\section{Introduction}

The iteration procedure is basically used in double bootstrap method to construct confidence interval in situation where the uncertainty estimation is questionable. Firstly introduced by Efron in 1983 [1], basically, the procedure is considered to use the plug-in method in each set of sample sequences of first bootstrap, and the replication size is done using small number than first replication. As proven by [2]-[6], this procedure eventually helps to increase the accuracy estimation which is focusing on the confidence interval estimation.

Despite of its great advantage in improving the first bootstrap accuracy estimation, the procedure seems to demand a high computer processing and eventually provide a 
range of time to complete the whole algorithm of iteration [2] [7]-[9]. Motivated by these problems, this paper proposes an alternative procedure of double bootstrap method which aims to increase the accuracy estimation by constructing the interval and reduce the computer processing. The proposed method considers a large sampling sequence of double bootstrap replication where the replication size is obtained and from approximation bias estimation method; refer to [10].

Instead of directly do the sampling procedure on sample data, this paper is motivated to conduct a hybrid modelling procedure, which is considered an example model of exponentially weighted moving average and hybrid it with proposed double bootstrap method. Next, the performance of this hybrid model is valued upon its accuracy estimation by constructing two-sided Studentised confidence interval [4] [11]. In terms of sample data, three set data from Gaussian distribution are generated in Monte Carlo simulation study and a set of real data of sukuk Ijarah from Bursa Malaysia are considered in this research. Generally, the sukuk Ijarah is a branch of Islamic investment certificate which is prohibited the element of usury, uncertainty (gharar) and gambling along its financial process.

Thus in this paper, Section 2 presents a discussion on the example model and purposed double bootstrap method. Next, Section 3 presents the sukuk Ijarah sample data and the number of double bootstrap replication that obtained from using sukuk data. In Section 4, the performance evaluation of hybrid model is presented and Section 5 is for research conclusion.

\section{Methodology}

Let $\varpi$ denote exponentially weighted moving average model. Consider that $x_{i}$ is independent variable and $\lambda$ is desirable parameter of $0 \leq \lambda \leq 1$ which could be known as decay factor of $\varpi$ model. T thus the one-step-ahead of $\varpi$ estimation can be referred to following recursion:

$$
\begin{gathered}
\hat{\varpi}_{i}=\lambda x_{i}+(1-\lambda) \hat{\varpi}_{i-1} \\
x_{i}=\hat{\varpi}_{i-1}+\xi_{i}, \quad i=1, \cdots, n
\end{gathered}
$$

where $\xi_{i}$ is a white noise process and the one-step-ahead residual estimation of Equation (1) can be made using formula of $\hat{\varepsilon}_{i}=x_{i}-\hat{\varpi}_{i-1}$.

The one-step-ahead of $\hat{\varepsilon}$ is implemented to residual double bootstrap scheme. In this research, an alternative double bootstrap sampling that proposed by [12] is applied. Note that, basically the bootstrapping method required a replication size for sampling with replacement procedure. As proposed by [10], a bootstrap replication size can be obtained by finding the converge value of bootstrap bias estimation. Motivating by this study, a simple modification has been made. Let a true estimator denote as $\eta_{0}$ and $\hat{\eta}$ be expected estimation of $E(\eta)$ which engaged from Equation (1). Thus, the bias estimation can be referred to following recursion:

$$
\tau=\frac{\sum_{i=1}^{b}\left[E(\eta)-\eta_{i}\right]}{b}, \quad i=1, \cdots, b ; b=1, \cdots, B B ;
$$


where $b$ denotes as current bootstrapping replication size, and note that two bootstrap procedure are used in this research, thus the respective bias estimation for single and double bootstrap are denotes as $\tau^{b}$ and $\tau^{b b}$. The rational replication size is obtained when Equation (3) is shown to be converged at certain value. By using this result, it can further be used in bootstrapping procedure.

First procedure is to sample the one-step-ahead of $\hat{\varepsilon}$ with replacement to obtained a set of single bootstrap independent variable, $x^{b}$. The first sampling is completed using rational size of replication from calculating the $\tau^{b}$ of Equation (3). Once the $x^{b}$ is obtained, the one-step-ahead of single bootstrap model and its residual can be estimated using respective formula of $\hat{\omega}_{i}^{b}=\lambda x_{i}^{b}+(1-\lambda) \hat{\omega}_{i-1}^{b}$ and $\hat{\varepsilon}_{i}^{b}=x_{i}^{b}-\hat{\omega}_{i-1}^{b}$.

Consider to draw the $\hat{\varepsilon}_{i}^{b}$ randomly to obtained a set of double bootstrap independent variable sample, $X_{i}^{b b}=\left\{x_{1}^{b b}, \cdots, x_{m}^{b b}\right\}$, where $m=1, \cdots, b b$. The draw is completed using second rational replication size, $\tau^{b b}$ that obtained by using Equation (3). Once the second sampling procedure is completed, the one-step-ahead double bootstrap model is estimated using formula of $\hat{\varpi}_{i}^{b b}=\lambda x_{i}^{b b}+(1-\lambda) \hat{\varpi}_{i-1}^{b b}$ with same parameter value used in Equation (1) and single bootstrap model.

This research is motivated to examine the efficiency of $\hat{\varpi}^{b b}$ estimation. Thus, by considering two-sided Studentised confidence interval of $\hat{\omega}^{b b}$ estimation, the efficiency can be obtained by finding the length of this interval. The estimation of double bootstrap confidence interval can be referred to following recursion:

$$
C I_{t^{b b}}=\left[\hat{\varpi}^{b b}-t_{1-\alpha} \hat{\sigma}\left(\hat{\varpi}^{b b}\right), \hat{\varpi}^{b b}-t_{\alpha} \hat{\sigma}\left(\hat{\varpi}^{b b}\right)\right]
$$

where $\hat{\sigma}\left(\hat{\varpi}^{b b}\right)$ denote as standard deviation of $\hat{\omega}^{b b}$ and $\alpha$ is considered to be $90 \%$ and $95 \%$ critical value of Studentised interval. Note that, the whole steps in this section has repetition algorithm and a computer programming is needed to do this simple calculation and looping command. Thus, in this research, it is motivated to use $\mathrm{R}$ language programming software to do the sampling procedure, estimate the models and its efficiency estimation.

\section{Materials}

A set sample data with respect to sukuk of Ijarah instrument investment issued by Malaysia Airports Capital Berhad is applied in this research. The selected instrument is coded as VN100268 under Islamic Medium-Term Notes Programme starting from 2010 until its maturity year of 2015 . For this research, the available daily price that has been issued is taken from October 08, 2010 until October 28, 2015 with sample size of 300. This sample data is plotted in Figure 1.

Based on Figure 1, the historical information price of sukuk Ijarah has shown to be increasing starting from its issuance in 2010 and slightly decrease in early 2011. The challenging year of 2011 gave a great impact of Malaysia Airports Capital Berhad trading activities which result to be its worst price issued on its historical trading. Learned by previous market trend, positive activities recorded along 2012 until early 2014 . However, a slow activity is shown in 2014 and 2015. 


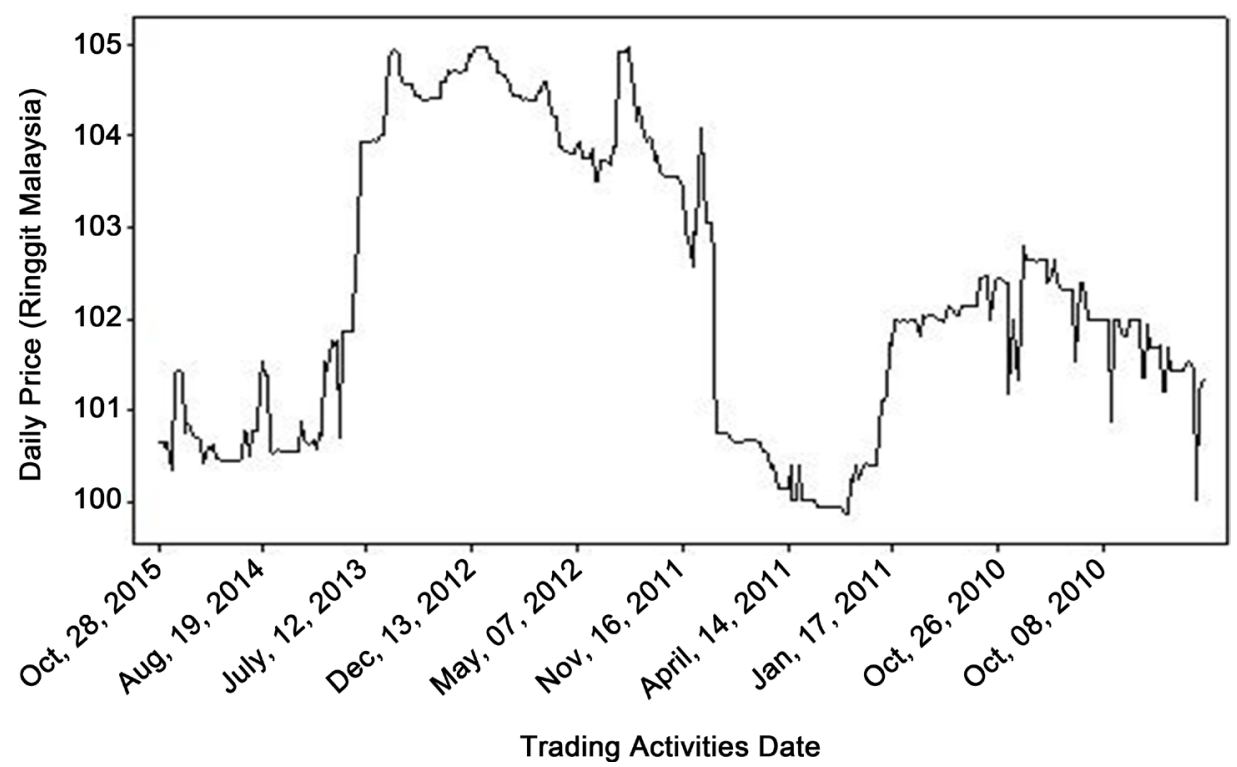

Figure 1. Daily price of sukuk Ijarah starting from 08 October 2010 until 28 October 2015.

For further step in estimating volatility of sukuk, a set of daily return sample is obtained using the formula proposed by [13]-[15]. By using this returns sample, the parameter of Equation (1) can be calculated using the proposed method by [16] [17]. Having a complete information needed to estimate one-step-ahead of Equation (1), further steps is to find the replication size of single and double bootstrap using the respective $\tau^{b}$ and $\tau^{b b}$. Even though the sample size of sukuk Ijarah has found to be greater compare to basic bootstrap conditional sample size, the bootstrap procedure could still be proceed because the method can helps to increase the efficiency of estimation [18]-[20]. Considering this advantage, thus, the tested replication size starts from 50 replications until the converged estimation value is obtained.

Table 1 shows bias estimation results for finding the rational single bootstrap replication size. It is found that the $\tau^{b}$ estimation is inconsistent at lower replication size, which is at 50 until 1700. However, an interesting result shown that the $\tau^{b}$ estimation starts to approach a certain value at 1740 until 1742 replications. By considering the greater number of this result [21], the rational replication number of single bootstrap procedure is 1742 .

Table 2 shows the bias estimation for rational double bootstrap replication size. Based on this table, it is found that the convergence starts at size of 2100 until 2200. Thus, based on this result and it is consider to choose a lower $\tau^{b b}$ estimation which is at 2150 to be used in double bootstrapping procedure.

\section{Performance Evaluation and Comparison}

In this section, the performance evaluation is based on estimation efficiency of single and double bootstrap model using the $90 \%$ and $95 \%$ of two-sided Studentised confidence interval. Instead of directly applied on daily return of sukuk Ijarah sample data, this research is motivated to firstly test the comparison of one-step-ahead $\hat{\omega}^{b}$ and 
Table 1. Bias estimation for single bootstrapping replication size.

\begin{tabular}{cc}
\hline Replication size & $\tau^{b}$ \\
1730 & 2.68 \\
1731 & 56.47 \\
1732 & 72.05 \\
1733 & 12.90 \\
1734 & 37.48 \\
1735 & 72.53 \\
1736 & 60.36 \\
1737 & 78.13 \\
1738 & 24.83 \\
1739 & 73.26 \\
1740 & 38.70 \\
1741 & 44.30 \\
1742 & 44.06
\end{tabular}

Table 2. Bias estimation for double bootstrapping replication number.

\begin{tabular}{cc}
\hline Replication size & $\tau^{b b}$ \\
1600 & 10.72 \\
1650 & 9.20 \\
1700 & 9.32 \\
1750 & 16.52 \\
1800 & 0.80 \\
1850 & 15.12 \\
1900 & 5.06 \\
1950 & 23.08 \\
2000 & 25.86 \\
2050 & 4.22 \\
2100 & 6.16 \\
2150 & 5.55 \\
2200 & 5.78 \\
\hline
\end{tabular}

$\hat{\varpi}^{b b}$ efficiency in Monte Carlo simulation study. The aim of this test is to study the efficiency pattern of both models in findings best estimator to be applied in real situation sample data.

\subsection{Monte Carlo Simulation}

Three sample size, $\left\{n: n_{1}, n_{2}, n_{3}\right\}$ are generated randomly from Gaussian distribution, $X_{n} \sim N(\mu, \sigma)$ using zero mean and one standard deviation. The generated samples 
are $\left\{n: n_{1}=30, n_{2}=60, n_{3}=200\right\}$ which is mostly used by [22] [23] for examine the pattern result when dealing with different size of sample, which is small, medium and large sample size. In terms of parameter value, consider a set of $\{\lambda: 1.00,0.90,0.75$, $0.50,0.40,0.30,0.25,0.10,0.05,0.03\}$ to be used in completing the estimation of one-step-ahead $\hat{\omega}^{b}$ and $\hat{\omega}^{b b}$. These values are motivated by the research of [24], [25]. According to their research, these parameter value are categorical as greater value, means that $\lambda=1.00$ down to $\lambda=0.30$, while the small value is from $\lambda=0.25$ until $\lambda=$ 0.03 . The aim of using different parameter values is examine the $\hat{\omega}^{b}$ and $\hat{\omega}^{b b}$ performance when the parameter is not estimated. The hybrid model performance is said to be efficient as the length of confidence interval approaching to true value as the sample size increase. By using all the information given, thus, the Studentised confidence interval can be estimated and its corresponding result can be referred to Table 3 and Table 4.

In Table 3 and Table 4, it shows the comparison of Studentised confidence interval estimation for one-step-ahead of single, $\hat{\omega}^{b}$ and double bootstrap model, $\hat{\omega}^{b b}$. Note that, at sample size of 30 , both of models seem to have decreasing length and the estimations are found to be shorter when using smaller $\lambda$. This indicates that both models are efficient when involving smaller sample size. Note that, for sample size of 60 , both models has shown inconsistent estimation when using $\lambda=0.40$ down to $\lambda=0.25$. For example $\hat{\omega}^{b}$ of $90 \%$ confidence interval, the length estimations are starts from $\{0.21053 \rightarrow 0.12977 \rightarrow 0.14599\}$. This indicates that both models are not well efficient using medium parameter value. However, an interesting result is found in double bootstrap model, $\hat{\omega}^{b b}$ where the length is continues decrease as $\lambda$ decrease, compare to single bootstrap.

Moreover, the length estimation of both models is shown to be consistently decreased as sample size increase. For example $\hat{\omega}^{b b}$ and $\lambda=0.90$, the estimation $90 \%$

Table 3. Comparison of $90 \%$ of two-sided studentised confidence interval.

\begin{tabular}{ccccccc}
\hline$n$ & 30 & 60 & 200 & 30 & 60 & 200 \\
\hline$\lambda$ & & $\hat{\omega}^{b}$ & & & $\hat{\omega}^{b b}$ & \\
\hline $1.00^{\mathrm{a}}$ & 0.45482 & 0.37090 & 0.23611 & 0.45442 & 0.37048 & 0.23594 \\
0.90 & 0.44995 & 0.35816 & 0.19873 & 0.40602 & 0.33016 & 0.18110 \\
0.75 & 0.45857 & 0.31597 & 0.17269 & 0.33434 & 0.26841 & 0.14280 \\
0.50 & 0.30310 & 0.18869 & 0.15291 & 0.24392 & 0.13324 & 0.12054 \\
0.40 & 0.27233 & 0.21053 & 0.11017 & 0.17472 & 0.16511 & 0.08060 \\
0.30 & 0.26264 & 0.12977 & 0.08115 & 0.17114 & 0.09140 & 0.05664 \\
$0.25^{\mathrm{b}}$ & 0.23160 & 0.14599 & 0.08457 & 0.14489 & 0.09815 & 0.05494 \\
0.10 & 0.09262 & 0.08606 & 0.04572 & 0.06577 & 0.03387 & 0.02724 \\
0.05 & 0.04530 & 0.03444 & 0.02669 & 0.01629 & 0.01061 & 0.01028 \\
0.03 & 0.03662 & 0.02391 & 0.02041 & 0.01186 & 0.01017 & 0.00835 \\
\hline
\end{tabular}

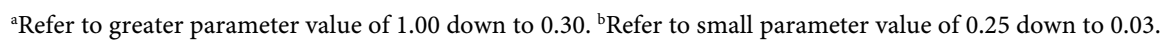


Table 4. Comparison of $95 \%$ of two-sided studentised confidence interval.

\begin{tabular}{ccccccc}
\hline$n$ & 30 & 60 & 200 & 30 & 60 & 200 \\
\hline$\lambda$ & & $\hat{\omega}^{b}$ & & & $\hat{\omega}^{b b}$ & \\
\hline $1.00^{\mathrm{a}}$ & 0.54746 & 0.44412 & 0.28174 & 0.54698 & 0.44362 & 0.28154 \\
0.90 & 0.54160 & 0.42886 & 0.23714 & 0.48872 & 0.39534 & 0.21611 \\
0.75 & 0.55198 & 0.37834 & 0.19757 & 0.40244 & 0.32140 & 0.17040 \\
0.50 & 0.30310 & 0.22594 & 0.18247 & 0.29360 & 0.15954 & 0.14384 \\
0.40 & 0.32780 & 0.25209 & 0.11017 & 0.21031 & 0.19771 & 0.09618 \\
0.30 & 0.31104 & 0.12977 & 0.09684 & 0.20600 & 0.10945 & 0.06759 \\
$0.25^{\mathrm{b}}$ & 0.27877 & 0.17481 & 0.10092 & 0.17440 & 0.11753 & 0.06556 \\
0.10 & 0.11149 & 0.10305 & 0.05456 & 0.07917 & 0.04056 & 0.03251 \\
0.05 & 0.05453 & 0.04124 & 0.03184 & 0.01961 & 0.01271 & 0.01226 \\
0.03 & 0.04408 & 0.02863 & 0.02435 & 0.01428 & 0.01218 & 0.00996 \\
\hline
\end{tabular}

a,b The abbreviation can be refer to Table 3 .

Table 5. Studentised confidence interval using sukuk Ijarah sample data.

\begin{tabular}{cccc}
\hline & \multicolumn{2}{c}{ Confidence Interval } & Length \\
\hline 90\% CI: & Lower & Upper & \\
\hline (a) Single Bootstrap Model & & & \\
(b) Double Bootstrap Model & -218.746 & 273.413 & 492.159 \\
$95 \%$ CI: & -191.359 & 267.963 & 459.322 \\
(a) Single Bootstrap Model & & & \\
(b) Double Bootstrap Model & -223.961 & 268.942 & 492.903 \\
\hline
\end{tabular}

and 95\% interval estimation are respectively start from $\{0.40602 \rightarrow 0.33016 \rightarrow 0.18110\}$ and $\{0.48872 \rightarrow 0.39534 \rightarrow 0.21611\}$. Note that, the estimation value of $\hat{\varpi}^{b}$ is also found to be shorter compare to $\hat{\varpi}^{b b}$. These results indicate that $\hat{\bar{\omega}}^{b b}$ is more efficient estimator compare to $\hat{\varpi}^{b}$.

\subsection{Example of Real Sample Data}

The daily return sample data of sukuk Ijarah is used to estimate the single and double bootstrap model. In this estimation procedure, the rational parameter value is found to be 0.94 . By using the same replication number of single and double bootstrap sampling procedure, the length of Studentised confidence interval could be proceed to be estimated. Thus, the result can be fevered to Table 5 .

Based on Table 5, it is shown that by using double bootstrap model as the estimator for Studentised interval, the interval length is found to be shorter compare to single bootstrap. For example 90\% confidence interval, the respective estimation of $\hat{\omega}^{b}$ and $\hat{\bar{\omega}}^{b b}$ is shown to be 492.159 and 459.322 . Note that, by using a critical value of $\alpha=0.05$, the length estimation is found to give greater value compare to $\alpha=0.10$. 


\section{Conclusion}

In this paper, an alternative procedure of double bootstrap was proposed and considered to be hybrid into an example model. The performance of this hybrid model was tested in terms of its accuracy estimation in simulation study and a real sample data known as sukuk Ijarah. A large sampling sequence of double bootstrap was found to shorter the Studentised interval estimation when using small range parameter of example model in simulation study. The length estimation found to be shorter as sample size increased. Moreover, for sukuk Ijarah sample data, it was shown that double bootstrap model has the shortest confidence interval compared to single bootstrap model. Based on both studies, it can conclude that large sampling sequence of double bootstrap has increased the accuracy estimation and improved the single bootstrap estimation.

\section{Acknowledgements}

A special gratitude for School of Informatics and Applied Mathematic (SIAM) and Research Management Centre (RMC), Universiti Malaysia Terengganu for supported this research paper.

\section{References}

[1] Efron, B. (1990) More Efficient Bootstrap Computations. Journal of the American Statistical Association, 85, 79-89. http://links.jstor.org/sici?sici=0162-1459\%28199003\% http://dx.doi.org/10.1080/01621459.1990.10475309

[2] Martin, M.A. (1990) On the Double Bootstrap. Technical Report No. 347.

[3] Vinod, H.D. (1995) Double Bootstrap for Shrinkage Estimators. Journal of Econometrics, 68, 287-302. http://dx.doi.org/10.1016/0304-4076(94)01639-H

[4] Nankervis, J.C. (2005) Computational Algorithms for Double Bootstrap Confidence Intervals. Computational Statistics and Data Analysis, 49, 461-475. http://dx.doi.org/10.1016/j.csda.2004.05.023

[5] Buckley, C. and Carney, P. (2013) The Potential to Reduce the Risk of Diffuse Pollution from Agriculture While Improving Economic Performance at Farm Level. Environmental Science and Policy, 25, 118-126. http://dx.doi.org/10.1016/j.envsci.2012.10.002

[6] Chang, J. and Hall, P. (2015) Double-Bootstrap Methods That Use a Single Double-Bootstrap Simulation. Biometrika, 102, 203-223. http://dx.doi.org/10.1093/biomet/asu060

[7] Hall, P. and Martin, M.A. (1988) On Bootstrap Resampling and Iteration. Oxford Journal, 75, 661-671. http://www.jstor.org/stable/2336307 http://dx.doi.org/10.1093/biomet/75.4.661

[8] Teyarachakul, S., Chand, S. and Tang, J. (2006) Estimating the Limits for Statistical Process Control Charts: A Direct Method Improving Upon the Bootstrap. European Journal of Operational Research, 178, 472-481. http://dx.doi.org/10.1016/j.ejor.2006.01.038

[9] Nedelea, I.C. and Fannin, J.M. (2013) Technical Efficiency of Critical Access Hospitals: An Application of the Two-Stage Approach with Double Bootstrap. Health Care Management Science, 16, 27-36. http://dx.doi.org/10.1007/s10729-012-9209-8

[10] Akhmad, F., Ibrahim, N.A., Isa, D. and Mohd. Rizam, A.B. (2003) Interval Selisih Rata-Rata dengan Metode Bootstrap Persentil. Jurnal Teknik Industri, 8, 110-117. 
[11] Pascual, L., Romo, J. and Ruiz, E. (2006) Bootstrap Prediction for Returns and Volatility in GARCH Models. Computational Statistic \& Data Analysis, 50, 2293-2312. http://dx.doi.org/10.1016/j.csda.2004.12.008

[12] Nurul Hila, Z. and MuhamadSafiih, L. (2016) The Performance of BB-MCEWMA Model: Case Study on Sukuk Rantau Abang Capital Berhad, Malaysia. International Journal of Applied, Business and Economic Research, 2, 639-653.

[13] Schwartz, A.R. and Whitcomb, K.D. (1977) Evidence on the Presence and Cause of Serial Correlation in Market Model Residuals. The Journal of Finance and Quantitative Analysis, 12, 291-313. http://www.jstor.org/stable/2330436 http://dx.doi.org/10.2307/2330436

[14] Laurence, M., Cai, F. and Qian, S. (1997) Weak-Form Efficiency and Causality Test in Chinese Stock Market. Multinational Finance Journal, 1, 291-307. http://dx.doi.org/10.17578/1-4-3

[15] Okpara, G.C. (2010) Stock Market Prices and the Random Walk Hypothesis: Further Evidence from Nigeria. Journal of Economics and International Finance, 2, 49-57.

[16] Cox, D. R. (1961). Prediction by Exponentially Weighted Moving Averages and Related Methods.Journal of the Royal Statistical Society, Series B (Methodological), 23(2): 414-422.

[17] Ramjee, R., Crato, N. and Ray, B.K. (2002) A Note Moving Average Forecasts of Long Memory Processes with an Application to Quality Control. International Journal of Forecasting, 18, 291-297. http://dx.doi.org/10.1016/S0169-2070(01)00159-5

[18] Chen, B., Gel, Y.R., Balakrishna, N. and Abraham, B. (2011) Computationally Efficient Bootstrap Prediction Intervals for Returns and Volatilities in ARCH and GARCH Processes. Journal of Forecasting, 30, 51-71. http://dx.doi.org/10.1002/for.1197

[19] Pascual, L., Romo, J. and Ruiz, E. (2006) Bootstrap Prediction for Returns and Volatility in GARCH Models. Computational Statistic \& Data Analysis, 50, 2293-2312. http://dx.doi.org/10.1016/j.csda.2004.12.008

[20] Nedelea, I.C. and Fannin, J.M. (2013) Technical Efficiency of Critical Access Hospitals: An Application of the Two-Stage Approach with Double Bootstrap. Health Care Management Science, 16, 27-36. http://dx.doi.org/10.1007/s10729-012-9209-8

[21] Efron, B. and Tibshirani, R.J. (1993) An Introduction to the Bootstrap. Chapman \& Hall, New York, London. http://dx.doi.org/10.1007/978-1-4899-4541-9

[22] Serel, D. A. and Maskowitz, H. (2008) Joint Economic Design of EWMA Control Charts for Mean and Variance. European Journal of Operational Research, 184, 157-168. http://dx.doi.org/10.1016/j.ejor.2006.09.084

[23] Tosasuku, J., Budsaba, K. and Volodin, A. (2009) Dependent Bootstrap Confidence Intervals for a Population Mean. Thailand Statistician, 7, 43-51.

[24] Lucas, J.M. and Saccucci, M.S. (1990) Exponentially Weighted Moving Average Control Schemes: Properties and Enhancements. Technometrics, 32, 1-12. http://dx.doi.org/10.1080/00401706.1990.10484583

[25] Montgomery, D.C. and Mastrangelo, C.M. (1991) Some Statistical Methods for Autocorrelated Data. Journal of Quality Technology, 23, 179-204. 
Submit or recommend next manuscript to SCIRP and we will provide best service for you:

Accepting pre-submission inquiries through Email, Facebook, LinkedIn, Twitter, etc. A wide selection of journals (inclusive of 9 subjects, more than 200 journals)

Providing 24-hour high-quality service

User-friendly online submission system

Fair and swift peer-review system

Efficient typesetting and proofreading procedure

Display of the result of downloads and visits, as well as the number of cited articles

Maximum dissemination of your research work

Submit your manuscript at: http://papersubmission.scirp.org/

Or contact ojs@scirp.org 